\title{
Benefícios do protocolo de manuseio mínimo em pacientes pré-termos extremos: percepção da equipe multiprofissional
}

\author{
Advantages of minimum handling protocol in patients prematures extreme: multiprofissional \\ team's perception \\ Beneficios del protocolo de manejo mínimo en pacientes pretérminos extremos: percepción del \\ equipo multiprofesional
}

Andreize de Freitas Ramos ORCID: https://orcid.org/0000-0002-0765-2787 Santa Casa de Misericórdia de Porto Alegre, Brasil E-mail: afr.enf@rede.ulbra.br

Sheila Beatris Kochhann ORCID: https://orcid.org/0000-0002-0841-0931 Santa Casa de Misericórdia de Porto Alegre, Brasil E-mail: Sheila_kochhann@hotmail.com

Jonas Hantt Corrêa Lima

ORCID: https://orcid.org/0000-0001-5672-1024 Universidade Luterana do Brasil, Brasil E-mail: jonashantt@hotmail.com

Bruna Reis Krug

ORCID: https://orcid.org/0000-0002-7811-6957 Universidade Luterana do Brasil, Brasil E-mail: brunakrug1606@gmail.com

Isabella Salzano Marchese

ORCID: https://orcid.org/0000-0001-5179-0311 Universidade Luterana do Brasil, Brasil E-mail: isabella.smarchese@gmail.com

Giulia Parmeggiani Zampieri

ORCID: https://orcid.org/0000-0002-5566-6307 Universidade Luterana do Brasil, Brasil E-mail: giuliazampp@gmail.com

Alana Zanella

ORCID: https://orcid.org/0000-0002-7736-9442 Universidade Luterana do Brasil, Brasil E-mail: alana.zanella@ rede.ulbra.br

Tales Antunes Franzini

ORCID: https://orcid.org/0000-0002-0148-6730 Universidade Federal de Ciências da Saúde de Porto Alegre, Brasil E-mail: talesafranzini@gmail.com

Vitória Fassina

ORCID: https://orcid.org/0000-0003-2962-9737

Universidade Luterana do Brasil, Brasil E-mail: vitoria_fassina@hotmail.com

Carlos Hugentobler Gonçalves

ORCID: https://orcid.org/0000-0001-5668-071X

Universidad María Auxiliadora, Paraguai

E-mail: carlos_hug@outlook.com

Thamela Gazola Zanatta

ORCID: https://orcid.org/0000-0002-6638-9843 Universidade Luterana do Brasil, Brasil

E-mail: thamelagazolazanatta@yahoo.com.br

Victória Machado Scheibe

ORCID: https://orcid.org/0000-0003-2033-9805 Universidade Luterana do Brasil, Brasil

E-mail: vick.machado.scheibe@ gmail.com

Manoela Sauer Faccioli

ORCID: https://orcid.org/0000-0002-9739-1372 Universidade Luterana do Brasil, Brasil E-mail: manoelafaccioli@gmail.com 


\begin{abstract}
Resumo
O protocolo considera o princípio de Levine, a respeito da conservação de energia, integridade estrutural, pessoal e social, proporcionando suporte terapêutico com menos estresse para RNs de alto risco. A pesquisa objetiva conhecer a compreensão dos profissionais envolvidos na sistematização do Protocolo de Manuseio Mínimo (PMM) e investigar, no olhar da equipe, a influência do PMM na vida atual e a longo prazo no RN prematuro extremo durante a assistência, conhecer como é constituído o PMM na instituição e descrever os sentimentos dos profissionais quanto à capacitação para as boas práticas da realização adequada do método. Trata-se de uma pesquisa descritiva com abordagem qualitativa. Foi realizada com a equipe multiprofissional da Unidade de Terapia Intensiva Neonatal (UTIN), do Hospital Municipal de Canoas/RS. Os profissionais entrevistados foram médicos, fisioterapeutas, enfermeiros e profissionais formados em nível técnico de enfermagem que utilizaram em algum momento o PMM no RN extremo ou de alto risco. A coleta de dados deu-se através de uma entrevista semiestruturada com cinco perguntas abertas, que formaram as pré-categorias: constituição do PMM na instituição, benefícios a curto prazo e longo prazo, capacitação para a realização do manuseio mínimo. Os critérios de exclusão foram os profissionais desse hospital que recusaram-se a participar ou ausentavam-se. O principal benefício encontrado no trabalho foi a prevenção de hemorragia craniana através do PMM e do baixo estímulo foto, todavia evidenciou-se o indicie aquém do necessário de treinamentos da equipe. O presente trabalho foi aprovado pelo Comitê de Ética sob parecer no 3.361 .742 e CAAE 09495519.4.0000.5349.
\end{abstract}

Palavras-chave: Recém-nascido; Unidades de terapia intensiva neonatal; Enfermagem neonatal.

\begin{abstract}
The protocol considers Levine's principle, regarding energy conservation, structural, personal and social integrity, providing therapeutic support with less stress for high-risk NBs. The research aims to understand the understanding of professionals involved in the systematization of the Minimum Handling Protocol (MMP) and investigate, in the team's view, the influence of the PMM in the current and long-term life of extreme premature NBs during care, to know how it is constituted the PMM in the institution and describe the professionals' feelings regarding training for good practices for the proper performance of the method. This is a descriptive research with a qualitative approach. It was carried out with the multidisciplinary team of the Neonatal Intensive Care Unit (NICU), of the Municipal Hospital of Canoas/RS. The professionals interviewed were physicians, physiotherapists, nurses and professionals trained at the technical level of nursing who at some point used the PMM in extreme or high-risk NBs. Data collection took place through a semi-structured interview with five open questions, which formed the pre-categories: constitution of the PMM in the institution, short-term and long-term benefits, training to perform the minimum handling. The exclusion criteria were the professionals from that hospital who refused to participate or were absent. The main benefit found in the work was the prevention of cranial hemorrhage through the PMM and the low photo stimulus, however, the index was found to be below the necessary training for the team. The present work was approved by the Ethics Committee under opinion $\mathrm{n}^{\circ} 3.361,742$ and CAAE 09495519.4.0000.5349.
\end{abstract}

Keywords: Newborn; Neonatal intensive care units; Neonatal nursing.

\title{
Resumen
}

El protocolo considera el principio de Levine, en cuanto a conservación de energía, integridad estructural, personal y social, brindando apoyo terapéutico con menor estrés a los $\mathrm{RN}$ de alto riesgo. La investigación tiene como objetivo comprender la comprensión de los profesionales involucrados en la sistematización del Protocolo Mínimo de Manejo (MMP) e investigar, en opinión del equipo, la influencia del PMM en la vida actual y a largo plazo de los RN prematuros extremos durante el cuidado, para conocer cómo se constituye el PMM en la institución y describir el sentir de los profesionales en cuanto a la formación de buenas prácticas para el correcto desempeño del método. Se trata de una investigación descriptiva con enfoque cualitativo. Se realizó con el equipo multidisciplinario de la Unidad de Cuidados Intensivos Neonatales (UCIN), del Hospital Municipal de Canoas / RS. Los profesionales entrevistados fueron médicos, fisioterapeutas, enfermeros y profesionales capacitados en el nivel técnico de enfermería que en algún momento utilizaron el PMM en RN extremos o de alto riesgo. La recolección de datos se realizó a través de una entrevista semiestructurada con cinco preguntas abiertas, que conformaron las pre-categorías: constitución del PMM en la institución, beneficios a corto y largo plazo, capacitación para realizar el manejo mínimo. Los criterios de exclusión fueron los profesionales de ese hospital que se negaron a participar o estuvieron ausentes. El principal beneficio encontrado en el trabajo fue la prevención de la hemorragia craneal a través del PMM y el bajo fotoestímulo, sin embargo, el índice se encontró por debajo del entrenamiento necesario para el equipo. El presente trabajo fue aprobado por el Comité de Ética con el dictamen no 3.361.742 y CAAE 09495519.4.0000.5349.

Palabras clave: Recién nacido; Unidades de cuidados intensivos Neonatales; Enfermería neonatal.

\section{Introdução}

O nascimento de um bebê passa por fases que envolvem tanto questões emocionais quanto fisiológicas. As mudanças fisiológicas são naturais e necessárias para a adaptação extrauterina, por isso são de extrema importância. Não raro o milagre 
do nascimento pode sofrer interferência de questões que antecipem o momento, levando o recém-nascido (RN) a uma internação inesperada na Unidade de Terapia Intensiva Neonatal (UTIN) (Costenaro, 2017).

No Brasil, a taxa de prematuridade vem aumentando; em 2016, o país apresentou uma taxa de 9,2\% de nascidos prematuros (Ribeiro, Silva, Santos, Luz, \& Coêlho, 2016). Em 2017, esse número saltou para 11,5\%, sendo o dobro dos países europeus. Destes, cerca de 26\% são pré-termos extremos (São Paulo, 2015; Fundação Oswaldo Cruz, 2016). Neste contexto, a utilização do protocolo de manuseio mínimo tornou-se elementar para o cuidado com o pré-termo extremo.

O conceito atual que conhecemos de Unidade de Terapia Intensiva (UTI) vem da época de Florence Nightingale, que utilizou as triagens e a vigilância constante em seu processo de trabalho (Golinelli \& Viana, 2017), sendo a UTI caracterizada como uma área distinta nos hospitais com maior aporte tecnológico (Biodi \& Ribeiro, 2014). Não haveria melhor local para os RNs de alto risco serem mantidos sob vigilância constante. Para o planejamento de uma UTIN deve ser levado em conta os avanços tecnológicos para o cuidado exclusivo do RN, pois ruídos, iluminação intensa e os procedimentos invasivos afetam o desenvolvimento cerebral, ocular e auditivo do RN (Tamez, 2017).

Relacionado ao nascimento do recém-nascido pré-termo (RNPT) decorrente de uma gestação normal, sabe-se que é idiopático, ou seja, não tem uma etiologia definida, mas sofre influência de inúmeros fatores, que podem interromper esta gravidez prematuramente, causando a maioria das internações da UTIN (Wilson \& Dockenberry, 2014). Os principais fatores que podem interferir na gestação são os fatores maternos, sejam socioeconômicos, tais como: desnutrição, idade e etnia; patológicos, como doenças cardíacas, renais, Diabetes mellitus tipo I ou II, hipertensão arterial sistêmica, ou comportamentais, bem como: abuso de substâncias, tabagismo, ausência ou deficiência no cuidado pré-natal (Wilson \& Dockenberry, 2014).

Perante o conceito de prematuridade e dos potenciais agravos que um nascimento prematuro pode causar em um RN, e sabendo que a assistência a esses pacientes não se limita exclusivamente a alta hospitalar, como também a uma melhor qualidade de vida; o suporte adequado aos RNs torna-se um fator desafiador quando não se tem o devido conhecimento (Naka, 2017). Nesse cenário, a capacitação dos profissionais que atuam diretamente com os RNPT é de extrema necessidade, a educação permanente que mistura ao dia a dia dos serviços e procedimentos o ensinar é um excelente método para capacitar os profissionais, devido ser uma medida que traz motivação para a aplicação de novas práticas (Costa, 2014).

Estudos, em 1970, mencionavam que a quantidade de manuseio de um RN está diretamente relacionada com o desenvolvimento ou com o agravo do quadro do paciente. Em 1990, foi citado pela primeira vez o protocolo para enfermeiros de UTI, onde é estabelecida a correlação do gasto de energia dos pacientes pelo toque do profissional (Langer, 1990). O protocolo leva em consideração o princípio de Levine, a respeito da conservação de energia, integridade estrutural, pessoal e social, proporcionando um suporte terapêutico com menos estresse para RNs de alto risco (Langer, 1990).

O manejo mínimo $(\mathrm{MM})$ no neonato se refere à manipulação mínima no RN prematuro extremo. Essa manipulação deve ser multiprofissional, para que seja garantido um número mínimo de estimulação (Giodani, Berte, \& Loureiro, 2017). Também é o ato de dar segurança e conforto ao RN, delimitando seu espaço (Martin, 2014). Os seus principais objetivos seriam a minimização do estresse ocasionado pelo manuseio demasiado e diminuir os possíveis agravos decorrentes do toque excessivo (Beleza \& Chagas, 2014).

Os neonatos quando são confrontados com uma situação estressora seja ela real ou percebida desencadeiam uma série de eventos tanto de nível neurológico como endócrino que precisam ser resolvidos, tal situação, acaba levando a um gasto de energia que deveria ser direcionado para os tecidos vitais, foco, atenção e memória, mas acabam sendo gastas para manter a homeostase corporal para que, por fim, sejam depositadas no desenvolvimento do RN (Raineki, Lucion, \& Weinberg, 2014).

Este cuidado envolve não somente o toque do profissional, mas também as condições que levam ao estresse do prematuro. Para isso, o cuidado com a iluminação, com os ruídos e com a posição do RNPT extremo é levado em consideração, para que esses fatores estressores não atrasem o desenvolvimento do RN (Tamez, 2017). 
O Protocolo de Manuseio Mínimo (PMM) foi implantado nas UTINs com o objetivo de reunir os procedimentos que serão realizados durante o turno, a fim de que o neonato tenha condições de ter quietude, sem exaustões desnecessárias, visando única e exclusivamente a melhora do paciente. Viu-se a necessidade da implantação do PMM nas UTINs em razão de ser uma técnica barata e que demanda tão somente a organização da equipe multiprofissional (Giordani, Berte, \& Loureiro, 2017).

Em decorrência de o protocolo ser considerado uma soft skill, ferramenta leve, que não demanda tecnologias pesadas, mas sim uma organização da equipe multiprofissional percebeu-se a necessidade de visualizar pela ótica dos profissionais quais os benefícios encontrados na realização do protocolo, como eles acreditam que a aplicação desse método de cuidado vai influenciar na qualidade de vida desses recém-nascidos pré-termos extremo.

\section{Metodologia}

Trata-se de uma pesquisa de caráter descritivo exploratório, com abordagem qualitativa. A coleta de dados deste estudo foi realizada de 01 de junho a 01 de julho de 2019, participaram do estudo 16 profissionais diretamente envolvidos com o MM de uma unidade neonatal em um hospital universitário, localizado no município de Canoas, Rio Grande do Sul.

A unidade neonatal desta instituição de saúde dispõe de trinta e cinco leitos, sendo vinte leitos destinados aos cuidados intensivos, dez leitos de cuidados intermediários, e cinco conjuntos leito materno/berço do recém-nascido em Alojamento Canguru, onde são atendidos pacientes do Sistema Único de Saúde (SUS) e outros convênios. É a 27ª instituição reconhecida pelo Ministério da Saúde como Centro de Referência para o método Canguru no país.

Para determinar o número de participantes, utilizou-se, além dos critérios de inclusão e exclusão, o princípio de saturação de dados. A amostra é, portanto, estabelecida por saturação de dados onde ocorre que, em certo ponto não se encontram novas informações, alcançando-se então a redundância (Polit, Beck, \& Hungler, 2011).

Foram adotados como critérios de inclusão: Profissionais graduados em medicina, fisioterapia e enfermagem e profissionais formados em nível técnico de enfermagem que participaram em algum momento da aplicação do protocolo de manejo mínimo no recém-nascido extremo ou de alto risco. Os critérios de exclusão foram os profissionais desse hospital que se recusaram a participar ou os ausentes no dia da pesquisa.

O "corpus" de análise do estudo foi formado pelos dados obtidos através de entrevistas semiestruturadas com perguntas fechadas relacionadas aos antecedentes profissionais, com objetivo de caracterizar a amostra do estudo, e cinco perguntas abertas.

As entrevistas foram realizadas em um espaço cedido pela instituição e em todos os turnos de trabalho (matutino, vespertino, noite 1 e noite 2). Não houve tempo preestabelecido para a realização da entrevista. As entrevistas foram gravadas e, posteriormente transcritas e analisadas. Os resultados serão divulgados somente para fins de pesquisa e serão guardados por um período de cinco anos, sendo esses destruídos posteriormente.

Os dados foram analisados por meio da técnica de análise de conteúdo com o comprimento das três etapas: préanálise, exploração do material, tratamento dos dados e interpretação (Minayo, 2013).

Seguindo os aspectos éticos, formulou-se o Termo de Consentimento Livre e Esclarecido (TCLE), constituído de duas vias de igual teor, sendo uma destinada à autora e a outra ao sujeito da pesquisa, assegurando o anonimato deles, a participação espontânea e o uso das informações para fins de pesquisa. Para proteção e sigilo do anonimato dos participantes, estes foram identificados com codinomes. Os participantes do estudo foram descritos pelas iniciais de sua graduação ou curso seguido do numeral conforme sequência de resposta da classe, por exemplo, M1 (médico), E1 (enfermeiro), F1 (fisioterapeuta) e TE1 (técnico de enfermagem).

A pesquisa atendeu à Resolução n 196/96 (Brasil, 1996), do Conselho Nacional de Saúde, e obteve Parecer da 
Comissão Ética em Pesquisa em Seres Humanos (CEP) da Universidade Luterana do Brasil, sob o número de parecer 3.361.742 e CAAE 09495519.4.0000.5349.

\section{Resultados e Discussão}

Dos profissionais que foram entrevistados individualmente, em um tempo médio de 30 minutos cada, oito são do sexo feminino e duas do sexo masculino. Da distribuição de profissionais entrevistados temos: dois da categoria médica, três fisioterapeutas, dois técnicos de enfermagem e três enfermeiros.

É importante ressaltar o período em que estavam inseridos no serviço, sendo que o menor período é de dois meses no serviço e o máximo 10 anos trabalhando na Unidade de Terapia Intensiva Neonatal (UTIN). A idade dos entrevistados variou entre 28 e 40 anos.

Referente a atuarem em Neonatologia anteriormente em outra instituição, somente quatro funcionários relataram ter trabalhado em Neonatologia, somente um profissional referiu que a antiga instituição não tinha implementado o protocolo de manuseio mínimo.

A partir da análise realizada emergiram cinco categorias: Constituição do protocolo de manuseio mínimo na instituição, benefícios do protocolo, dividido em duas subcategorias: curto e longo prazo, capacitação da equipe e reestruturação do protocolo. O surgimento destas categorias resultou na discussão apresentada a seguir.

\subsection{Constituição do Protocolo de Manuseio Mínimo na Instituição}

Com o avanço do conhecimento e o desenvolvimento tecnológico, observa-se o emprego crescente de tratamentos invasivos e cuidados médicos e de enfermagem intensivos para manter a vida de RN gravemente enfermos. Se, por um lado, tais tratamentos mantêm os bebês vivos, por outro lado ocasionam, muitas vezes, dor e sofrimento aos mesmos, desta forma deve-se buscar medidas de proteção aos riscos e desconfortos ocasionados aos RNs em tratamento nas UTINs (Brasil, 2014).

O prematurinho extremo que é 96 horas sem manusear, sem pesar, sem movimentar, sem lateralizar, e depois quando fica maior, é uma vez por turno. Só mexe uma vez e não mexe mais. Seria o ideal, né, mas a gente sabe que não, né, porque são os prematuros que precisam de mais [manuseio e cuidado], tem coleta, raio-x, então acaba às vezes sendo o mais manuseado, essa é a verdade. (TE1).

Além disso, o enfermeiro é responsável por promover a adaptação do RN ao meio externo através de medidas como manutenção do equilíbrio térmico, adequação da umidade, luminosidade, ruídos e estímulos; acompanhamento do quadro clínico, sinais vitais e desenvolvimento; atender às necessidades do RN; elaborar e implementar um plano de cuidados; coordenar e supervisionar a assistência de enfermagem ao RN e família (Ribeiro, Silva, Santos, Luz, \& Coêlho, 2016).

Vale ressaltar a fala do profissional, a seguir:

Bebês abaixo de $1 \mathrm{~kg}$ são de manuseio exclusivo do enfermeiro, sendo manuseados uma vez ao turno. Então verificamos pressão, HGT, troca de fraldas, verifica sinais vitais, faz todos os cuidados com este bebê. Acompanhamos a físio durante a aspiração e durante a fisioterapia motora, é tudo o enfermeiro quem faz. (E1)

\subsection{Benefícios do Protocolo de Manuseio Mínimo}

$\mathrm{O}$ protocolo pode ser utilizado para determinada patologia ou condição, como também pode abranger um todo. $\mathrm{O}$ Hospital Southern Regional Medical Center situado no estado da Georgia, Estados Unidos, utiliza o método para prevenção de hemorragias intracraniana, que é uma situação que tinha recorrência no hospital e eles viram no protocolo de toque mínimo uma forma para diminuir os índices de incidência dessa condição (Tamez, 2017). 
A resposta do organismo ao estresse controlada pelo sistema nervoso autônomo e a resposta aos estímulos ambientais são imaturas nos RNPT e quaisquer intervenções e o próprio ambiente da UTIN são agentes estressores. Assim, é necessário analisar a adequação das intervenções a fim de não sobrecarregar e prejudicar a homeostase orgânica, com risco de agravos à condição clínica da criança e prolongar o período de internação (Freitas \& Kimura, 2019).

\section{Benefícios a curto prazo}

O recém-nascido (RN) de alto risco possui instabilidade fisiológica e hemodinâmica, que geralmente são ocasionadas pela gestação de alto risco, tendo como conseqüência distúrbios congênitos, alterações metabólicas, prematuridade, asfixia perinatal e problemas durante a gravidez, necessitando de cuidados especializados em UTIN após o nascimento (Pereira, Câmara, \& Pereira, 2019).

Cabe o apontamento do profissional abaixo:

Influencia em tudo, o bebe que é manuseado está instável o tempo inteiro, os nossos bebês do manuseio mínimo eles são os menores e os mais graves. Então o desfecho a longo prazo, a médio prazo e a curto prazo vai depender desse primeiro manuseio. Então influencia em tudo. (F1)

Apesar dos crescentes avanços na assistência ao recém-nascido ao longo das últimas décadas, em virtude do surgimento de novas tecnologias e da organização de UTIN, a exposição dos RN a essas tecnologias, associada a manipulações excessivas, pode trazer prejuízos importantes ao seu crescimento e desenvolvimento (Frank, Quaresma, \& Silva, 2019).

Ressalta-se a afirmativa seguinte a seguir captada em entrevista:

Acho que influencia 100\%, principalmente nos nossos pacientes, quanto menos você estressar e estressar significa até mesmo abrir a porta da incubadora, mais saúde e a recuperação deles é mais rápida, com menos sequelas. Então o protocolo é a parte mais importante do nosso trabalho. (M1)

Em relação aos estímulos visuais, é importante em ambientes como a UTIN, que haja uma redução da luminosidade, favorecendo uma melhora na qualidade do sono, diminuição do estresse e estimulação ao ganho de peso. A claridade excessiva pode trazer alterações fisiológicas e bioquímicas, podendo afetar o desenvolvimento normal da retina dos prematuros e causar cegueira (Ramos, Rezio, Peixoto, \& Gardenghi, 2016).

Pontua-se abaixo importante ponto de vista a ser lembrado:

Em tudo, porque ele recém saiu do útero, tu pega um prematurinho extremo ali de 500-400g, né, e qualquer barulho pra eles, qualquer barulho pra eles já causa uma hemorragia cerebral, pode dar um dano irreversível. (TE2)

\section{Benefícios a longo prazo}

Estudos que medem a emotividade, reatividade hormonal relacionada ao estresse sugerem que o tratamento melhora a capacidade de lidar com esse estresse, melhorando também a adaptação com o meio e, por fim, resultando em um organismo emocionalmente estável, para um desenvolvimento adequado (Raineri, Lucion, \& Weinberg, 2014).

Prestemos atenção a precisão técnica evidenciada na sentença:

Desenvolvimento neurossensorial é o benefício fundamental porque ele ainda tem às suturas abertas então ele ainda pode desenvolver uma hemorragia crânio encefálica, então a gente interfere muito nisso a longo prazo, questão motora do bebê, a gente ajuda muito ele, faz o manuseio com delicadeza, questão pulmonar normalmente esse prematuro está intubado porque é $R N$ extremo, então a gente tem esse cuidado para que o paciente não seja extubado, por extubação acidental que às vezes ocorre quando o técnico manuseia e acaba não tendo essa destreza e cuidado e o bebê acaba tendo riscos para extubação de repetição então nós temos um cuidado maior. (E2) 
Os neonatais quando são confrontados com uma situação estressora seja ela real ou percebida desencadeiam uma série de eventos tanto neurológicos como endócrinos para que se possam ser resolvidas, levando a um gasto de energia que deveria ser direcionado para os tecidos vitais, foco, atenção e memória, mas que acabam sendo gastas essas energias para ter uma homeostase corporal para que, por fim, sejam depositadas no desenvolvimento do RN (Raineri, Lucion, \& Weinberg, 2014).

Ressaltando mais um ponto de vista profissional:

Diminuição de sequelas neurológicas é a principal. Diminuição do catabolismo, ou seja, perda fisiológica de peso, então quando menos a gente estressa, porque tudo gira em torno do estresse, mais rápido ele recupera e isso tem uma influência depois na primeira infância, com a recuperação nutricional e o aumento de defesas, combate às infecções. Garante um aconchego maior ao bebê porque tem a questão do ninho, do calor da incubadora então isso também tem um benefício no psicológico da criança. E o principal é isso: a diminuição do comprometimento neuropsicomotor na infância posterior. (M2)

\subsection{Capacitação da Equipe}

Para a implantação de um protocolo ou uma técnica nova precisa-se ter conhecimento do saber prévio dos colaboradores para que seja então escolhido o material educacional, porque a partir da vivência de cada um deles associando-se a uma literatura pode-se desenvolver um método prático e adequado ao cuidado voltado para o paciente (Souza \& Ribeiro, 2017).

Pontua-se o discurso:

Sim, eu ajudei a elaborar o protocolo (F2).

Nesse contexto, a educação continuada se faz necessária, tanto por cursos como por reuniões com interesse no debate de assuntos como também uma atualização contínua de interesse dos próprios profissionais (Martin, 2014).

Apontamento abaixo:

Não, a gente falou em grupo, mas não foi feito nada assim, como faz como não faz. (M1)

Durante a entrevista evidenciou-se a confusão de um colaborador quanto à diferença entre o que seria o protocolo de manuseio mínimo e o método canguru.

Referindo a entrevista a seguir:

Sim, sim a gente faz o método canguru que é adotado aqui na instituição. (TE2)

Este método se divide em duas etapas basicamente: Na primeira etapa, preconiza-se acesso precoce e livre dos pais à UTIN, estímulo à amamentação e participação da mãe nos cuidados do bebê, bem como início do contato pele a pele logo que as condições clínicas do bebê permitam. Na segunda etapa, mãe e bebê permanecem em enfermaria conjunta, e a posição canguru deve ser realizada pelo maior tempo possível (Carvalho, Maia, \& Costa, 2018).

Para que tenha uma melhor qualidade no tratamento realizado ao paciente tem-se a necessidade do investimento em recursos quanto à educação e treinamento dos profissionais atuantes no cuidado (Brandão \& Fernandes, 2018). No Brasil, devido ao número reduzido de revistas e de congressos na área da educação em saúde, falar sobre capacitação aos profissionais tornou-se imperativo. Os centros educacionais têm se inovado para criarem oportunidades de ensino adequado para o treinamento dos profissionais, mas ainda muito aquém do que o necessário (Brandão \& Fernandes, 2018). 


\subsection{Reestruturação do Protocolo}

Os bebês são manipulados para higiene, para a verificação dos sinais vitais e os procedimentos programados pela equipe médica. Também circula um número significativo de profissionais que participam de visitas, round e outras atividades dentro da unidade (Coelho et al., 2018).

Ressalte e perceba a afirmativa na construção do conhecimento prático e relacional abaixo:

Mudaria, como nós somos um hospital escola, a gente tem um pouco de dificuldade de fazer com que a equipe médica se organize e mexa menos nesse paciente. Eu não usaria horários fixos, né, esperaria a demanda do paciente, ah ele vai ter uma coleta, ele vai ter um exame, então vamos esperar o momento em que vão ser feitos esses procedimentos e então a gente faz o cuidado desse paciente. (F3)

Cada profissional da equipe tem sua função no atendimento do paciente, e durante o trabalho em equipe nota-se a dificuldade nos diferentes ambientes de atenção à saúde, gerada principalmente pela deficiência de reflexões e discussões teórico-práticas no processo de formação acadêmica. Os estudos apontam, na sua maioria, um descompasso entre a formação acadêmica e a atuação na prática profissional. Nesse descompasso fica claramente evidente a fragmentação e a dissociação dos saberes disciplinares difundidos pela Universidade (Backes et al., 2014).

Segue fala pontual:

Sim, mas na verdade ele deveria ser mais maleável. Existem situações, por exemplo, que o bebê entra em protocolo e não pode ser manuseado excessivamente, mas é um bebê que está extremamente grave. Então isso faz com que a gente tenha que entrar mais e isso normalmente gera um conflito médico-enfermagem. (M2)

O protocolo em si é muito completo o que existe é, como tudo na vida, o ideal e o real, às vezes o paciente se desestabiliza e tu acaba tendo que quebrar esse protocolo várias vezes. (E3)

Nesse ínterim percebemos a importância de aprender alguns temas mais na prática para que possa ser exequível em realidades que não são sempre as ideais do contexto de um país como o nosso, com muitos recursos escassos. Profissionais capacitados conseguem conciliar teoria e prática e sabem, de modo geral, fornecer a melhor terapêutica adaptando ao seu meio laboral. Vale ressaltar, reconhecimento da liberdade e autonomia de todos os envolvidos no processo de pesquisa, inclusive da liberdade científica e acadêmica como destaca os preceitos éticos previstos nas Resoluções 466/2012 - 510/2016 - 580/2018, do Ministério da Saúde.

\section{Considerações Finais}

O presente estudo possibilitou compreender as diferentes percepções de benefícios que os profissionais acreditam obter através da adequada realização do protocolo de manuseio mínimo. Apesar das limitações do estudo no sentido de análise e captação dos profissionais que não responderem, não elencar categoricamente os motivos de recusa.

O principal benefício encontrado durante o trabalho foi a prevenção de hemorragia craniana, que, através do mínimo manuseio e os estímulos foto sensoriais, pode ser evitada com sucesso.

Quanto à capacitação dos profissionais evidenciou-se um déficit, pois dos dez entrevistados somente dois tinham conhecimento de um treinamento prévio para a realização do protocolo. O que se explica quando olhamos os períodos de serviço dos profissionais que não tiveram treinamento prévio, todos estavam no máximo um ano no setor de Neonatologia, por essa razão pode-se dizer que há um ano a instituição não fornece treinamento adequado ao profissional que exercerá o cuidado ao neonato prematuro extremo.

Viu-se que foi unânime a necessidade de adequação do protocolo a realidade do paciente pré-termo extremo, que normalmente é o paciente mais debilitado na UTIN. Adequar o protocolo às necessidades do serviço facilitará o atendimento 
multiprofissional, assim como suas relações interpessoais.

Uma das limitações desta pesquisa se deu frente à dificuldade de acesso aos profissionais devido à complexidade do cuidado com os pacientes internados na UTIN, conforme evidenciado no presente estudo.

Com objetivo de colaborar com o seguimento do protocolo de manuseio mínimo na instituição de saúde em que foi realizado o estudo, tem-se a ideia de levar um feedback frente aos resultados obtidos. Desta forma, espera-se contribuir para as boas práticas do manejo mínimo do neonato, de maneira a perpetuar os benefícios obtidos até o momento pela instituição e comprovados cientificamente. Novos estudos necessitam serem realizados na área para aprimorar e evoluir no tema

\section{Referências}

Backes, D. S., Carpes, A. D., Piovesan, C., Haeffner, L. S. B., Büscher, A., \& Lomba, L. (2014). Trabalho em equipe multiprofissional na saúde: da concepção ao desafio do fazer na prática. Disciplinarm Scientia: Ciências da Saúde, 15(2), 277-89.

Beleza, L., \& Chagas, A. C. C. (2014). Protocolo de manuseio mínimo. Brasília, DF: Hmib.

Biodi, R. S., \& Ribeiro, R. A. B. (2014). Critérios de admissão e alta em UTI. In: Guimarães HP, Assunção MSC. Manual de medicina intensiva: associação de medicina intensiva brasileira. São Paulo: Atheneu. Cap. 1, p. 1239.

Brandão, C. F. S., \& Fernandes, D. C. (2018). Importância e desafios do treinamento simulado em saúde. Scientia Medica, 28(1), $30102-06$.

Brasil. (1996). Resolução n 196, de 10 de outubro de 1996. Dispõe sobre a pesquisa envolvendo seres humanos. Brasília, DF: Conselho Nacional de Saúde.

Brasil. (2014). Ministério da Saúde. Secretaria de Atenção à Saúde. Departamento de Ações Programáticas Estratégicas. Atenção à saúde do recém-nascido: guia para os profissionais de saúde. ( $2^{\mathrm{a}}$ ed. Atual): Ministério da Saúde.

Carvalho, E. T. S., Maia, F. S., \& Costa, R. S. L. (2018). Método canguru: o papel do enfermeiro frente aos cuidados de enfermagem. Ciências em Foco, 2(2), 99-113.

Coelho, A. S., Custódio, D. C. G. G., Silva, J. S. C., Rosso, G., Silva, R., \& Carniel, F. (2018). Equipe de enfermagem e a assistência humanizada na UTI Neonatal. Re On Facema, 4(1), 873-7.

Costa, O. M. (2014). Intervenção educativa sobre hipotermia neonatal: uma estratégia para mudanças no cuidado da enfermagem. Monografia (Especialização) - Curso de Especialização em Linhas de Cuidado em Enfermagem - Opção Saúde Materna, Neonatal e do Lactente, Enfermagem. Florianópolis: Universidade Federal de Santa Catarina.

Costenaro, R. G. S. (2017). Há se eu pudesse falar. O que eu diria para quem me cuida? In: Costenaro, R. G. S., Corrêa, D. A. M., \& Ichisato, S. M. T. Cuidados de enfermagem em Neonatologia. Porto Alegre: Moriá; Cap. 1, p. 29-42.

Frank, E. S. M. P., Quaresma, A. C. M., \& Silva, J. S. N. (2019). O cuidado de enfermagem ao recém-nascido prematuro em unidade de terapia intensiva neonatal. Journal of Specialist, 3(3), 1-18.

Freitas, P., \& Kimura, A. F. (2019). Biomarkers of stress in preterm newborns exposed to bath: a pilot study. Online Brazilian of Journal Nurse, 17(1), 119-26.

Fundação Oswaldo Cruz. (2016). Taxa de bebês prematuros no país é quase o dobro do que em países da Europa. Rio de Janeiro: FioCruz.

Giordani, A. T. K., Berte, C., \& Loureiro, P. C. (2017). Cuidados essenciais com o prematuro extremo: elaboração do protocolo mínimo manuseio. Disciplinarm Scientia: Ciências da Saúde, 3(2), 165-72.

Golinelli, P. C., \& Viana, R. A. P. P. (2017). Unidade de Terapia Intensiva: planta física, organização e administração de recursos humanos e materiais. In: Viana, R. A. P. P., \& Torre, M. Enfermagem em Terapia Intensiva: práticas integrativas. Barueri: Manole, Cap. 1, p. 2-8.

Langer, V. (1990). Minimal handling protocol for the intensive care nursery. Neonatal Network, 9(3), 23-7.

Martin, S. H. (2014). Manipulação mínima do RN. Minas Gerais: Ipsemg.

Minayo, M. C. S. (2013). Pesquisa social: Teoria, método e criatividade. (33a . ed.): Vozes.

Naka, S. H. (2017). Controle térmico do recém-nascido prematuro na primeira semana de vida: o uso da incubadora umidificada. Dissertação (Mestrado) Curso de Pós-Graduação em Saúde da Criança e do Adolescente, Ciências da Saúde. Curitiba: Universidade Federal do Paraná.

Pereira, R. M. S., Câmara, T. L., \& Pereira, N. C. S. T. (2019). Enfermagem e o manuseio do recém-nascido na unidade de terapia intensiva neonatal. Revista Uningá, 56(2), 222-33.

Polit, D. F., Beck, C. T., \& Hungler, B. P. (2011). Fundamentos de pesquisa em enfermagem: métodos, avaliação e utilização. (7ª ed.): Artmed.

Raineki, C., Lucion, A. B., \& Weinberg, J. (2014). Neonatal handling: an overview of the positive and negative effects. Developmental Psychobiology, 56(8), $1613-25$.

Ramos, B. A., Rezio, G. S., Peixoto, F. A. O., \& Gardenghi, G. (2016). A importância da manipulação mínima em recém-nascidos prematuros na unidade de terapia intensiva neonatal. Revista Eletrônica de Saúde e Ciências, 6(2). 
Research, Society and Development, v. 10, n. 12, e160101217870, 2021

(CC BY 4.0) | ISSN 2525-3409 | DOI: http://dx.doi.org/10.33448/rsd-v10i12.17870

Ribeiro, J. F., Silva, L. L. C., Santos, I. L., Luz, V. L. E., \& Coêlho, D. M. M. (2016). O prematuro em unidade de terapia intensiva neonatal: a assistência do enfermeiro. Revista de Enfermagem da UFPE, Pernambuco, 10(10), 3833-41.

Secretaria de Saúde. Manual de Neonatologia. São Paulo: Secretaria de Saúde, p. 18-22.

Souza, G. S. L., \& Ribeiro, M. A. R. R. (2017). Construção de manual sobre cirurgia segura para profissionais de saúde. Cogitare Enfermagem, 22(1), 1-5.

Tamez, R. N. (2017). Enfermagem na UTI neonatal: assistência ao recém-nascido de alto risco. (6ª . ed.): Guanabara Koogan.

Wilson, D., \& Hockenberry, M. J. (2014). Wong: Fundamentos de enfermagem pediátrica. (9a. ed.): Elsevier. 